www.jmscr.igmpublication.org

Index Copernicus Value: 79.54

ISSN (e)-2347-176x ISSN (p) 2455-0450

crossref DOI: https://dx.doi.org/10.18535/jmscr/v7i4.171

\title{
Comparative assessment of Cartridge based Nucleic Acid amplification Technique with Microscopy among Pulmonary TB Patients in a Tertiary care Hospital of Tripura
}

\author{
Authors \\ Dr Pradip Kumar Das ${ }^{1}$, Dr. Niladri Sekhar Das ${ }^{2}$, Dr Jayanta Debnath ${ }^{3}$, \\ Dr Anup Saha ${ }^{2}$ \\ ${ }^{1}$ Professor, ${ }^{2}$ Assistant Professor, ${ }^{3}$ Associate Professor, \\ TMC and DR BRAM Teachinhg Hospital, Hapania Agartala, Tripura \\ *Corresponding Author \\ Dr Niladri Sekhar Das
}

TMC and DR BRAM Teaching Hospital, Hapania Agartala, Tripura, India

Phone No 8974816077, Email drniladrisekhar@gmail.com

\begin{abstract}
In the era of rapid diagnosis of TB and Drug resistance Cartridge Based Nucleic Acid Amplification Technique (CBNAAT) came with a future of early and easy diagnosis for TB. However Microscopic examination of sputum sample still is the most commonly used screening test and Lowenstein Jensen (LJ) culture method considered as a gold standard technique. Hence a study was planned to see the Concordance and efficacy of CBNAAT with smear Microscopy at TMC and Dr BRAM teaching Hospital Hapania Tripura from January to December 2017.

Material and Methods: CBNNAT and smear microscopy of 278 TB suspected patients were done ion the department of Microbiology.

Results: Smear positive cases CBNAAT positivity was $95.91 \%$ and smear negative cases $10.48 \%$. Concordance of two techniques (CBNAAT and Microscopy) for the diagnosis of Tuberculosis was noticed to be $90.64 \%$. $74.55 \%$ of cases were found to be negative by CBNAAT. Total 72 cases were positive for MTb and 4 cases were Rifampicin resistance.

Conclusion: CBNAAT positive result suggest but negative result do not exclude TB. CBNAAT does not eliminate the need of conventional microscopy however $L J$ should be establish soon that are required to monitor the CBNAAT negative cases and to detect resistance to drugs other than Rifampicin.

Keywords: CBNAAT, Sputum Microscopy, Drug resistance.
\end{abstract}

\section{Introduction}

Globally, India is a home for more than $25 \%$ of global Tuberculosis (TB) burden. The sensitivity of smear microscopy and its inability to detect drug resistance limits its impact for TB control. RNTCP was launched in 1997, which was formulated as the most systemic and Costeffective approach to revitalize the TB control programme in India ${ }^{[1]}$. Out of the estimated global annual incidence of 9 million TB cases, India alone shares the incidence of 2.1 million (24\%) cases/year (one fourth of global incidence). For 
the diagnosis of pulmonary TB Microscopic examination of Sputum sample is the most commonly used screening test however requires a large no of bacteria $\left(10^{4} / \mathrm{ml}\right.$ of sputum ${ }^{[2]}$ further it can't differentiate MTB from Non tubercular Mycobacteria (NTM). Lowenstein Jensen (LJ) culture plays an important role for detection of M.tuberculosis (MTB) and considered as a gold standard technique. It requires less no of bacteria 10-100 bacilli /ml for isolation, allows species identification and Drug Sensitivity Testing (DST) for detection of resistant strain like Multi Drug Resistant TB (MDR-TB) and Extensive Drug Resistant -TB (XDR-TB) but needs time. The national strategic plan of 2012-2017 was to achieve universal access of quality of TB diagnosis along with $90 \%$ success rate of all new patients ${ }^{[1]}$. Though there are different techniques for the diagnosis of TB but no single diagnostic test currently satisfies all the demands of "rapid", "affordable", and "easy". In the era of rapid diagnosis of $\mathrm{TB}$ and Drug resistance Cartridge Based Nucleic Acid Amplification Technique (CBNAAT) came with a future of early, affordable, easy diagnosis for TB. Diagnostic accuracy of CBNAAT for pulmonary TB has been reported high in literature ${ }^{[3]}$. CBNAAT works on the principle of real time PCR and reports can be given in two hrs. The specificity of this technique for detecting $\mathrm{TB}$ and Rifampicin resistance recorded to be $99 \%$ and $98 \%$ accordingly. Tuberculosis section of Microbiology at TMC routinely doing CBNAAT. Hence this study was planned to see the Concordance and efficacy of CBNAAT with smear Microscopy for the diagnosis of TB and detection of Mycobacterium tuberculosis among pulmonary cases at our set up along with future prospective of Solid culture establishment (LJ) for DST and Tuberculosis Research.

\section{Material and Methods}

We included all adult pulmonary TB patients (Suspected, Old, New, Defaulter etc) whose sample was sent to Mycobacteriology Lab for
CBNAAT from January to December 2017. We included cases of

a) Presumptive pulmonary tuberculosis cases.

1. Cough $>2$ weeks

2. Fever $>2$ weeks

3. Significant weight loss

4. Blood in sputum

5. Any abnormalities in chest radiography.

b) TB Treatment failure cases

c) Contact with known MDR TB case

d) Sputum positive and negative at diagnosis , Retreatment cases

e) Follow up sputum positive cases

f) Microbiologically confirmed TB patients.

However we excluded a) Individuals not fitting in the definition of suspected TB cases.

b) HIV TB co-infection.

CBNAAT was performed in the Department of Microbiology TMC, according to manufacturer instruction maintaining all aseptic precaution though Sputum smear microscopy was done on the same sample at DMC of the college. Quantity of the sample was confirmed to be $>5 \mathrm{ml}$. MTB bacterial load was estimated in terms of High, Medium and Low. Demographical and Treatment information history was collected from the clinical notes. Smear microscopy along with CBNAAT result was compared and assessed in Excel sheet for analysis.

\section{Results}

278 no of Study subjects were enrolled for the study in the department of Microbiology from January to December 2017. Numbers of males $(n=218)$ were more than females $(n=60)$. Out of 278 TB suspected patients smear microscopy was positive among $17.62 \%$ of cases where as CBNAAT was in $25.53 \%$ cases. Males showed higher no of positivity (Table1) and 31-45 yrs of age was found to be more affected by TB. While Analysing the test results by two different techniques it was found that in smear positive cases CBNAAT positivity was $95.91 \%$ and smear negative cases $10.48 \%$. Concordance of two techniques (CBNAAT and Microscopy) for the 
diagnosis of Tuberculosis was noticed to be $90.64 \%$ though two cases were smear positive and CBNAAT Negative and 24 cases were smear negative and CBNAAT positive. 74.55 of cases were found to be negative by CBNAAT. Total 72 cases were positive for MTb and 4 cases were Rifampicin resistance.

Table 1: Age wise Analysis of Positive cases By Microscopy and/or CBNAAT technique

\begin{tabular}{|l|c|c|}
\hline Age $(\mathrm{yrs})$ & Male & Female \\
\hline$<15$ & 2 & 1 \\
\hline $16-30$ & 7 & 8 \\
\hline $31-45$ & 17 & 3 \\
\hline $46-60$ & 17 & 2 \\
\hline$>61$ & 10 & 4 \\
\hline Total positives & 53 & 18 \\
\hline
\end{tabular}

Table 2: Comparison of two techniques (CBNAAT and Staining)

\begin{tabular}{|l|c|c|c|}
\hline $\begin{array}{l}\text { CBNNAT } \\
\text { results }\end{array}$ & Smear +ve & Smear -ve & Total \\
\hline Positive & 47 & 24 & 71 \\
\hline Negative & 2 & 205 & 207 \\
\hline Total & 49 & 229 & 278 \\
\hline \multicolumn{4}{|l|}{ Smear positivity $17.6 \%$, CBNAAT $25.5 \%$} \\
\hline
\end{tabular}

\section{Discussion}

Early diagnosis of TB is necessary to disrupt the disease transmission chain. Although $\mathrm{ZN}$ smear positive patients are considered highly infectious and being focused by most of clinicians. Smear negative patients are also reported to responsible for approximately $17 \%$ of transmission and its impact on public health could not be neglected ${ }^{[4]}$. This preliminary hospital based study was designed to evaluate the diagnostic yield of Cartridge Based Nucleic Acid Amplification Technique (CBNAAT) for the detection of Mycobacteria form Sputum samples and to compare it with AFB staining result. Since solid culture facility yet to be established in our medical college reliability of staining result was compared with CBNAAT technique. We analysed total 278 samples of TB cases (Old and new) by two methodology of diagnosis (Microscopy and CBNAAT). Majority of the subjects in our study were belong to the age group of 31-45yrs and males were more peredominant than females like other same type of study ${ }^{[5,6]}$.
CBNAAT is a simple bench top point of care diagnostic assay that can be performed with minimal training. The results are available within 2 hours, much earlier than the culture which usually takes days to come positive ${ }^{[7,8]}$. Numbers of studies have demonstrated the utility of CBNAAT in diagnosis of pulmonary tuberculosis [910,11,12]. Studies showed that CBNAAT assay of respiratory specimens had a pooled sensitivity of $89 \%$ (95\% CI: $85 \%-92 \%$ ) and specificity of $99 \%$ (95\% CI: $98 \%-99 \%$ ) in the diagnosis of pulmonary $\mathrm{TB}^{[13]}$. Smear positivity in present study was found to be $17.6 \%$ and positivity by CBNAAT was found to be $25.5 \%$ respectively. Lower smear positivity by ZN stain and higher MTB positivity by CBNAAT were demonstrated in various studies ${ }^{14}$. A wide range of $0-75 \%$ positivity for $\mathrm{ZN}$ smear has been reported in other studies ${ }^{15}$ however variation in positivity among different techniques may be due to inclusion criteria, expertise and laboratory settings. While Analysing the test results by two different techniques it was found that in smear positive cases CBNAAT positivity was $95.91 \%$ and smear negative cases $10.48 \%$. Smear negative TB is more difficult to treat due to delay in reaching definite diagnosis, in such cases new diagnostic approaches could be fruitful in early diagnosis and prompt treatment, hence preventing the patients becoming infectious for others. Numerous studies revealed that overall sensitivity and specificity of CBNAAT technique is $86.8 \%$ and specificity is $93.1 \%$ taking $\mathrm{LJ}$ as a gold standard method ${ }^{[16,17]}$. We found $74.5 \%$ of cases were negative by CBNAAT. Culture using LJ medium is method of choice for diagnosis of MTB and considered as gold standard in developing countries and its importance could not be neglected. In our setup there is no facility yet established for culture of MTB so the reasons of CBNAAT negativity could not be evaluate. Though a study conducted by Muhammad et $\mathrm{al}^{[18]}$ showed out of total 13 negative cases of gene-Expert $69.2 \%$ consist of MTB and 30.8\% consist of NTM on the basis of para-nitro-benzoic acid test. In our study two 
samples were found to be microscopy positive and CBNAAT negative. The probable reason for false negative result would be PCR inhibitors present in the assay pragya et al ${ }^{[6]}$ or it could be the NTM as we do not have the facility of Solid culture like LJ.CBNAAT preclude the rapid Rifampicin susceptibility along with MTB detection. We detected $25.89 \%(72 / 278)$ strains of MTB out of total symptomatic cases with $5.5 \%$ of Rifampicin resistance. The development of CBNAAT technique is considered an important breakthrough in the fight against TB. This assay can detects $M$. tuberculosis as well as mutation that confer Rifampicin resistance using three specific primers and five unique molecular probes with in less than 2 hours. But the adoption of this molecular technique does not eliminate the need for conventional TB culture and DST on solid LJ. False negative and false positive results ${ }^{[19,20]}$ with ATT treatment and discordant rifampicin resistance was noted while comparing with LJ. Conventional microscopy and LJ culture will remain essential for monitoring therapy and DST for anti-TB agents other than Rifampicin.

\section{Conclusion}

Molecular techniques have revolutionized the diagnosis of TB, MDR TB. The CBNAAT assay is a rapid and easy-to-perform fully automated Nucleic acid amplification test, which is extremely helpful in early diagnosis and to initiate the treatment of tuberculosis. It has a high capability to detect MTB complex DNA in AFB microscopy negative as well as positive samples. The assay correctly detects the information from the rpoB hot spot region regarding rifampicin resistance. But it should be remembered that CBNAAT positive result suggest but negative result do not exclude TB. CBNAAT does not eliminate the need of conventional microscopy, culture and anti-tubercular drug sensitivity on LJ that are required to monitor the progression of treatment and to detect resistance to drugs other than Rifampicin. However this small scale study gave us an suggestion to establish the gold standard technique (LJ) in our set up with existing microscopy and CBNAAT for better interpretation and diagnosis of TB.

\section{References}

1. Editoreal: Recent Changes in Technical and Operational Guidelines for Tuberculosis Control Programme in India - 2016: A Paradigm Shift in Tuberculosis Control. The Journal of Association of Chest Physicians, Jan-Jun 2017 , Volume 5, Issue 1.

2. R Dewan, S Anuradha, A Khanna, S Garg, S Singla, P Ish, S Agarwal,A Narayana H, M Hanif, H Singh, S Uppal. Role of cartridge-based nucleic acid amplification test (CBNAAT) for early diagnosis of pulmonary tuberculosis in HIV. JIACM 2015; 16(2): 114-7

3. Monika Agrawal, Ashish Bajaj, Vinay Bhatia, Sarjana Dutt. Comparative Study of GeneXpert with ZN Stain and Culture in Samples of Suspected Pulmonary Tuberculosis.JCDR2016;10(5):9-12

4. Mostaza J L, Garcia N, Fernandez S, Bahamonde A, Fuentes M I, Palomo M J. Analysis and predictor of delay in suspicion and treatment among hospitalized patients with pulmonary tuberculosis. An Med Interna. 2007; 24(10): 478-83.

5. Munir M K, Anwer N, Iqbal R, Nosheen S, Rehman S, Salam A A. Comparision of Genotype MTBDRplus testing kit with conventional method for drug susceptibility testing of isoniazid and rifampicin in tuberculosis patients. Pak J Med Res. 2014; 53 (2): 25-30

6. Pragya Rani, Anil Kumar Bilolikar, Vishala CSarma, Udayasri B, Sukrutha Gopal Reddy, Prasanna Lakshmi Kakarla et al. A comparative study of AFB smear and GeneXpert MTB/ RIF assay in pulmonary and extrapulmonary specimens and Detection of rifampicin resistance in a tertiary care hospital. Journal of Medical and scientific research.2017;5(4):115-120 
7. World Health Organization: Automated real-time nucleic acid amplification technology for rapid and simultaneous detection of tuberculosis and rifampicin resistance: Xpert mtb/rif system. Policy statement 2011. http://whqlibdoc.who. int/publications/2011/9789241501545_eng. pdf.

8. Van Rie A, Page-Shipp L, Scott L, Sanne I, Stevens W. Xpert(®) MTB/RIF for pointof-care diagnosis of TB in high-HIV burden, resource-limited countries: hype or hope? Expert Rev Mol Diagn. 2010;10(7):937-46.

9. Bowles EC, Freyée B, van Ingen J, Mulder B, Boeree MJ, van Soolingen D. Xpert MTB/RIF®, a novel automated polymerase chain reaction-based tool for the diagnosis of tuberculosis. Int J Tuberc Lung Dis. 2011;15:988-89.

10. Malbruny B, Le Marrec G, Courageux K, Leclercq R, Cattoir V. Rapid and efficient detection of Mycobacterium tuberculosis in respiratory and non-respiratory samples. Int J Tuberc Lung Dis. 2011;15:553-55.

11. Marlowe EM, Novak-Weekley SM, Cumpio J, Sharp SE, Momeny MA, Babst A, et al. Evaluation of the Cepheid Xpert MTB/RIF assay for direct detection of Mycobacterium tuberculosis complex in respiratory specimens. J Clin Microbiol. 2011;49:162123.

12. Miller MB, Popowitch EB, Backlund MG, Ager EP. Performance of Xpert MTB/RIF RUO assay and IS6110 real-time PCR for Mycobacterium tuberculosis detection in clinical samples. J Clin Microbiol. 2011;49: 3458-62.

13. Steingart KR, Schiller I, Horne DJ, Pai M, Boehme CC, et al. Xpert MTB/RIF assay for pulmonary tuberculosis and rifampicin resistance in adults. Cochrane Database Syst Rev. 2014; 21(1): CD009593.
14. Buchelli Rmirez H L, Gracia-Clemente M M, Alvarez-Alvarez C, Palacio-Gutierrez J J, Pando-Sandoval A,Gagatek S, AriasGuillen M, Quenzada-Loaiza C A, CasanClara. Impact of the Xpert MTB/RIF molecular test on the late diagnosis of pulmonary tuberculosis.Int $\mathrm{J}$ Tuberc Lung Dis. 2014;18(4): 435-37.

15. Kamboj S S, Goel M M, Tandon P I. Correlation study of histopathology and bacteriology in patients of tubercular lymphadenitis.Indian J Chest Dis Allied Sci. 1994; 36: 187-91.

16. Pierre Le Palud P, Cattoir V, Malbruny B, Magnier R, Campbell K, Oulkhouir Y, et al. Retrospective observational study of diagnostic accuracy of the Xpert巴 MTB/ RIF assay on fiberoptic bronchoscopy sampling for early diagnosis of smearnegative or sputum-scarce patients with suspected tuberculosis. BMC Pulm Med. 2014;14 (1):137.

17. Lee HY, Seong MW, Park SS, Hwang SS, Lee J, Park YS, et al. Diagnostic [22] accuracy of Xpert ${ }^{\circledR}$ MTB/RIF on bronchoscopy specimens in patients with suspected pulmonary tuberculosis. Int $J$ Tuberc Lung Dis. 2013;17(7):917-21.

18. Comparison of Ziehl Neelsen Microscopy with GeneXpert for Detection of MycobacteriumTuberculosis. Muhammad Kashif Munir, Sana Rehman, Muhammad Aasim, Rizwan Iqbal,Saqib Saeed. IOSRJDMS. 2015:14(11):56-60

19. Siddiqi S. H., Ruesch-Gerdes S. MGIT procedure manual for BACTEC MGIT 960 TB system. Becton Dickinson, Franklin Lakes, NJ. 2006

20. Williams D, Waguespack C, Eisenach K, Crawford J, Portaels F, Salfinger M et al. Characterization of Rifampin Resistance in Pathogenic Mycobacteria. Antimicrob Agents Chemother. 1994; 38: 2380-86. 